\title{
Modelling-based evaluation of the effect of quarantine control by the Chinese government in the coronavirus disease 2019 outbreak
}

\author{
Xinkai Zhou ${ }^{1 \dagger}$, Zhigui $\mathrm{Wu}^{1 \dagger}$, Ranran $\mathrm{Yu}^{1 \dagger}$, Shanni Cao ${ }^{1 \dagger}$, Wen Fang ${ }^{1}$, Zhen Jiang ${ }^{1}$, Fang Yuan ${ }^{1}$, \\ Chao Yan $^{1,2^{*}} \&$ Dijun Chen ${ }^{1,2^{*}}$ \\ ${ }^{1}$ State Key Laboratory of Pharmaceutical Biotechnology, School of Life Sciences, Nanjing University, Nanjing 210023, China; \\ ${ }^{2}$ Institute of Artificial Intelligence Biomedicine, Nanjing University, Nanjing 210023, China
}

Received April 10, 2020; accepted May 2, 2020; published online May 8, 2020

Citation: Zhou, X., Wu, Z., Yu, R., Cao, S., Fang, W., Jiang, Z., Yuan, F., Yan, C., and Chen, D. (2020). Modelling-based evaluation of the effect of quarantine
control by the Chinese government in the coronavirus disease 2019 outbreak. Sci China Life Sci 63, 1257-1260. https://doi.org/10.1007/s11427-020$1717-9$

\section{Dear Editor,}

The recent outbreak of novel coronavirus disease 2019 (COVID-19) has already become a global-scale epidemic. Since the case from China was reported on Dec 30th, 2019 (Huang et al., 2020; Li et al., 2020), the pathogen was soon identified as a new coronavirus on Jan 7th, 2020 and tentatively named 2019-nCOV (Huang et al., 2020) and later renamed SARS-CoV-2 by the International Committee on Taxonomy of Viruses. However, strict control measures, including the lockdown of Wuhan city did not take effect until Jan 23rd, by which time several million people have already travelled outside Wuhan for the upcoming Chinese New Year, spreading the disease to other areas of China. For the whole month of February, rigorous prevention and control measures were taken by the Chinese government and the whole country is literally in a lockdown state. The necessity of these strict measures has been questioned both domestically and internationally concerning the devastating effects on the global economy; and there is also severe debate over when these control measures, including travel bans and public gathering bans, should be lifted. However, the effectiveness of these strict control measures has not been sys-

$\uparrow$ Contributed equally to this work

*Corresponding authors (Dijun Chen, email: dijunchen@nju.edu.cn; Chao Yan, email: yanchao@nju.edu.cn) tematically evaluated using epidemiological data. In this study, we provided a retrospective modelling-based evaluation of the effectiveness of government control measures on COVID-19 in China, using the public data by the end of February 2020.

Since the impact of an epidemic depends on both the number of persons infected and the spectrum of clinical severity, we first analyzed the change of severity rate and the fatality rate over time. We found that both parameters remain unchanged over the investigated phase for each province and are not affected by the strictness of control measures (Figure S1 in Supporting Information), indicating that the total number of severe cases and deaths are largely determined by the total number of infections, thus supporting the importance and urgency of outbreak prevention and control of transmission. The fact that the severity and the fatality rate remain unchanged during the whole epidemic course suggests that the biology of the virus itself did not change over time; this is in line with genetic sequencing results that few mutations are identified among virus samples collected from different generations of patients (Ceraolo and Giorgi, 2020; $\mathrm{Lu}$ et al., 2020). The significantly higher reported fatality rate in Wuhan city and Hubei province compared to other regions are probably due to the shortage of medical supplies and the underestimation of total cases of infection.

Using an adjusted susceptible-exposed-infectious-recovered (SEIR) model, we fitted the public data by taking the 
effect of prevention policies into consideration and achieved an almost perfect fit for the real data (Supplementary Methods in Supporting Information). Key epidemiological parameters including incubation rate $(\sigma)$, infectious rate $(\beta)$ and recovered rate $(\gamma)$ were estimated in a dynamic manner (Figure 1A). As expected, the incubation rate $(\sigma)$ shows little to none change over time (Figure 1B and C). In this regard, the average duration of incubation $(1 / \sigma)$ is estimated to be 5.9 days based on data in Wuhan, which is generally in agreement with existing reports (Backer et al., 2020; Chan et al., 2020; Guan et al., 2020). While the recovered rate shows a slow but steady increase over time (Figure 1D), the infectious rate dramatically changed during the whole outbreak process (Figure 1B and E). Of note, the dynamic values of the infectious rate are within the range of the recent estimation by Yang et al. (Yang et al. 2020), suggesting that our approach for model parameter estimation is plausible. The national infectious rate clearly peaked around Jan 23rd, the day of Wuhan lockdown and has continued to drop ever since. This trend is more apparent when analyzing the data of Wuhan city alone (Figure 1E). There is a one-week gap between the peak day (around Feb 1st) and the day of Wuhan lockdown, which is mostly due to the delay effect of prevention interventions (Figure 1E). The curve for other cities displayed a continuous descending trend from the beginning of the available data point (around Jan 20th, the city of Nanjing was shown as an example in Figure 1E), when strict control measures have already been in place. In Wuhan, the
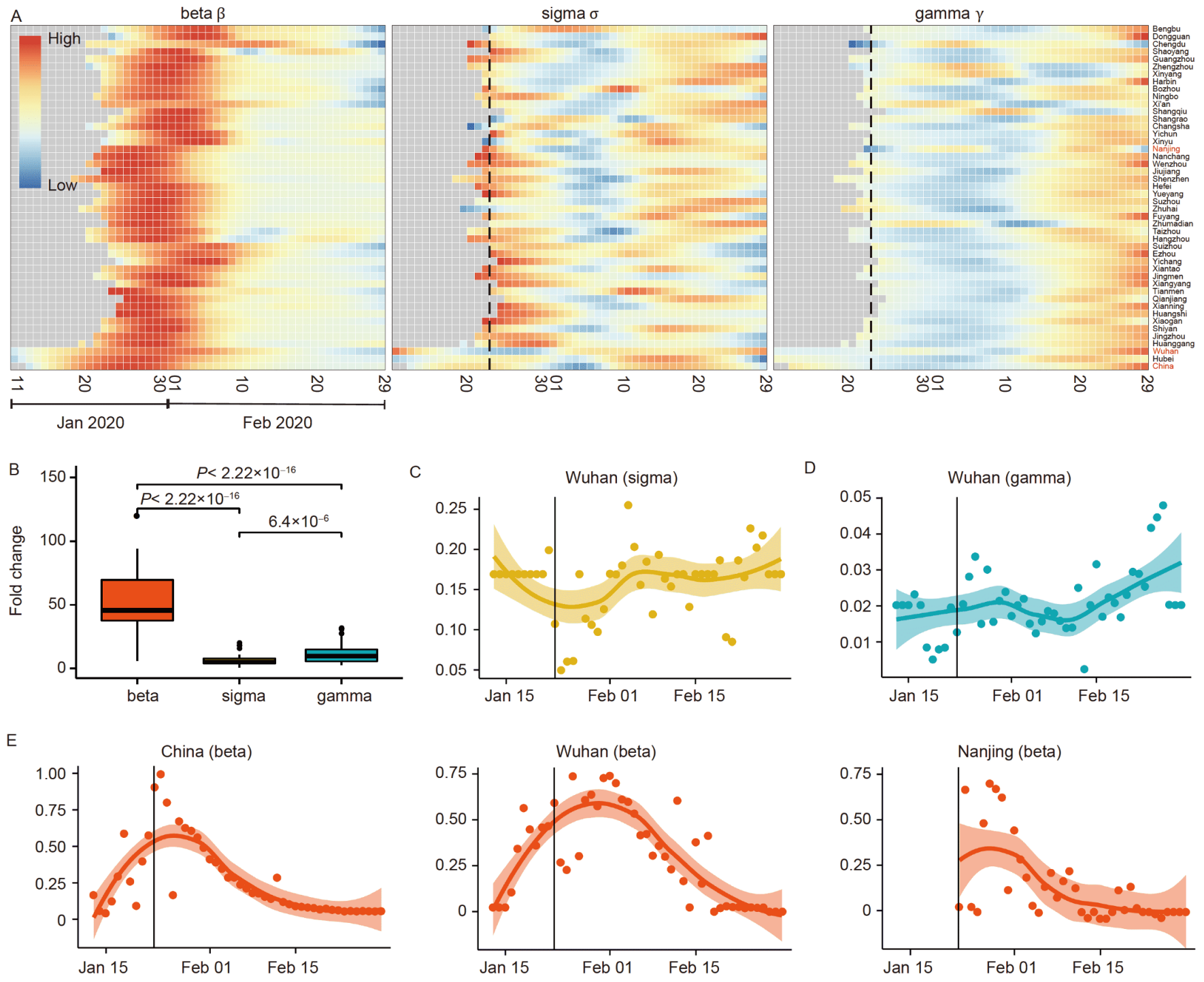

Figure 1 Parameter estimation of the SEIR transmission model. A, Heatmap showing the dynamic change of estimated parameters of the SEIR model over time. In order to compare data among different cities, only relative values are shown in the heatmap. Data for example cities (in red) are shown in C-E. B, Fold changes of the three parameters of the SEIR model. Dynamics of estimated $\sigma(\mathrm{C})$ or $\gamma$ (D) parameters over time in Wuhan. E, Dynamics of estimated $\beta$ parameter over time in the population of mainland China (left), Wuhan city (middle) and Nanjing city (right). In this figure, the modelling analysis was performed in the populations of the Hubei province, the mainland of China as well as the top 44 cities of the whole mainland of China. Dashed lines indicate the date of lockdown of Wuhan. 
recovery rate has continued to increase with more than 2-fold change which is probably due to the dramatic improvement of medical conditions, such as the immobilization of thousands of physicians from all over the country and immediate establishment of several new hospitals in Wuhan. These data indicate a significant correlation between the infectious rate and the recovery rate with prevention and control measures. To our knowledge, our work is the first to evaluate the effectiveness of government control on the spread of COVID19 using a modelling approach.

Next, we simulated the possible outcome scenarios with different degrees of government prevention. Assuming there is a direct correlation of the infectious rate $(\beta$ in the SEIR model) and the strictness of prevention measures, we simulated the outbreak dynamics in different prevention scenarios in Wuhan using different estimated infectious rate $(\beta)$ values while keeping other parameters $(\sigma$ and $\gamma)$ as their average values (Supplementary Methods in Supporting Information). In the case of low to no prevention (with high infectious rate at the early stage, from Jan 27th to Feb 1st as shown in Figure $1 \mathrm{E})$, the peak of predicted infection cases would eventually reach ca. 7.78 million, covering $70 \%$ of the whole population of Wuhan city (Figure S2A in Supporting Information). The number of potentially infected individuals dramatically reduces as the infectious rate decreases upon prevention interventions (after Feb 1st; Figure S2B in Supporting Information). These data suggest that the strictly enforced quarantine and travel ban have significantly decreased the otherwise uncontrollable spread of the disease.

By the end of February 2020, the COVID-19 epidemic has already been reported in more than 50 countries. Areas with a high risk of exponential outbreak include Japan, South Korea, Italy, Singapore and Iran. Since the situation in China has already been well controlled, a retrospective evaluation of the epidemiological characteristics and transmission dynamics would provide valuable insights that might help with disease control decisions worldwide. Using a similar approach, we next modeled all the publicly available data (by the end of February) on several different countries that already have considerable amounts of confirmed cases and predict their epidemic curve with various degrees of government control, assuming similar transmission properties of the virus in different counties (Figure S3 in Supporting Information). In Singapore, where only mild government intervention existed, a surprisingly continuous decrease in the infectious rate and increase in the recovery rate was observed, implying the potential inhibitory effect of warm climate on the spreading of the virus; whereas the situation in Italy, Korea, and Iran are quickly deteriorating. For Japan, although the infectious rate is getting better, the recovery rate is not, implying either a latency in hospitalization or lack of dedicated medical resources.

There is no doubt that the capability of management and control of COVID-19 transmission heavily rely on the preparedness of a country's health system. While it remains debatable whether large economy bodies in Asia such as South Korea and Japan should adopt similar control measures as the Chinese government; in less developed countries with insufficient medical resources and absence of a pandemic preparedness plan, a mild response might be inadequate to deal with such an outbreak. Extreme quarantine and transport control measures similar to China should be considered to mitigate local transmission following confirmed importation.

In conclusion, we have constructed an SEIR model and fitted all the publicly available China COVID-2019 data in a dynamic manner, and get a fairly accurate model of the whole process of the epidemic in China. We estimated the overall fatality rate to be $0.68 \%$ outside of Hubei province. Through simulation, we also evaluated the importance and effectiveness of strict government control enforced by Chinese authorities. It is clear that the control measures, both in Wuhan and nationwide, have significantly reduced transmissibility. Considering the potential threat of fast worldwide COVID-19 outbreak to public health and global economy, more strict government controls are advised based on the China experience.

Compliance and ethics The author(s) declare that they have no conflict of interest.

Acknowledgements We would like to thank Prof. Chenyu Zhang (Nanjing University, NJU) and Prof. Yu Xue (Huazhong University of Science and Technology, HUST) for their constructive discussion and suggestions. We also appreciate technical supports from Wanshan Ning (HUST) and Liang$\mathrm{Yu} F u$ (NJU). The study was supported by the Fundamental Research Funds for the Central Universities (14380129) and the National Natural Science Foundation of China (21877060).

\section{References}

Backer, J.A., Klinkenberg, D., and Wallinga, J. (2020). Incubation period of 2019 novel coronavirus (2019-nCoV) infections among travellers from Wuhan, China, 20-28 January 2020. Euro Surveill 25, pii: 2000062.

Ceraolo, C., and Giorgi, F.M. (2020). Genomic variance of the 2019-nCoV coronavirus. J Med Virol 92, 522-528.

Chan, J.F.W., Yuan, S., Kok, K.H., To, K.K.W., Chu, H., Yang, J., Xing, F., Liu, J., Yip, C.C.Y., Poon, R.W.S., et al. (2020). A familial cluster of pneumonia associated with the 2019 novel coronavirus indicating person-to-person transmission: a study of a family cluster. Lancet 395 , 514-523.

Guan, W.J., Ni, Z.Y., Hu, Y., Liang, W.H., Ou, C.Q., He, J.X., Liu, L., Shan, H., Lei, C.L., Hui, D.S.C., et al. (2020). Clinical characteristics of coronavirus disease 2019 in China. N Engl J Med 382, 1708-1720.

Huang, C., Wang, Y., Li, X., Ren, L., Zhao, J., Hu, Y., Zhang, L., Fan, G., $\mathrm{Xu}, \mathrm{J}$., Gu, X., et al. (2020). Clinical features of patients infected with 2019 novel coronavirus in Wuhan, China. Lancet 395, 497-506.

Li, Q., Guan, X., Wu, P., Wang, X., Zhou, L., Tong, Y., Ren, R., Leung, K. S.M., Lau, E.H.Y., Wong, J.Y., et al. (2020). Early transmission dynamics in Wuhan, China, of novel coronavirus-infected pneumonia. N Engl J Med 382, 1199-1207. 
Lu, R., Zhao, X., Li, J., Niu, P., Yang, B., Wu, H., Wang, W., Song, H., Huang, B., Zhu, N., et al. (2020). Genomic characterisation and epidemiology of 2019 novel coronavirus: implications for virus origins and receptor binding. Lancet 395, 565-574.
Yang, Z., Zeng, Z., Wang, K., Wong, S.S., Liang, W., Zanin, M., Liu, P., Cao, X., Gao, Z., Mai, Z., et al. (2020). Modified SEIR and AI prediction of the epidemics trend of COVID-19 in China under public health interventions. J Thorac Dis 12, 165-174.

\section{SUPPORTING INFORMATION}

The supporting information is available online at https://doi.org/10.1007/s11427-020-1717-9. The supporting materials are published as submitted, without typesetting or editing. The responsibility for scientific accuracy and content remains entirely with the authors. 